\title{
MATHEMATICAL OF TWO-DIMENSIONAL DYNAMIC DISTRIBUTION OF THERMAL AND PRODUCTION LOADS BETWEEN HEAT ELECTROPOWER STATION TURBINES
}

\author{
L.A. Belyaev" ${ }^{1, a}$, O.Y. Romashova ${ }^{1}$, M.A. Vagner ${ }^{1}$, S.A. Shevelev ${ }^{1}$, E. Skrebatun ${ }^{1}$ \\ ${ }^{1}$ National Research Tomsk Polytechnic University, 634050 Tomsk, Russia
}

\begin{abstract}
Two-dimensional dynamic programming algorithm for distribution of loads between the controlled heat electropower station (HES) extractions is developed. Main recurrent correlations of sequential optimization process by using modified Bellman criterion are presented. Results of program complex implementation are shown.
\end{abstract}

\section{Introduction}

Distribution of both electric and thermal loads between HES turbines is related to multi-dimensional optimization problem which is still not completely solved. The main reason is the complexity of energy characteristics (EC) of heat-extraction turbines together with the variety of modes and functioning conditions of main and auxiliary equipment. The dynamic programming method is considered to be universal optimization method suitable for any type of EC turbines. This method allows obtaining the single solution of a problem with defining objective function global minimum as well as possibility of taking into account any limitations for optimized variables. This method is successfully applied for distribution of electric load between condensation turbo-units of power stations and power system. The application of this method for HES mode optimization runs, however, into considerable calculation difficulties. These difficulties grow with the increase of number of independent parameters and load step decrease.

In order to obtain the HES global optimum one either applies complex load distribution with different optimization methods [1,2] or divides the initial problem into constituents [3]. Therefore the problem of thermal distribution between controlled turbines extractions frequently becomes independent within the boundaries of general HES modes optimization problem.

\section{Mathematical model and study technique}

Let us outline the mathematical description of two-dimensional dynamic programming main correlations for optimal distribution of both given production $D_{P \Sigma}$ and heating $D_{H \Sigma}$ loads of the station between n turbo-units of HES. For simplicity let us limit ourselves to turbine operation according to thermal schedule.

Minimum specific heat consumption for a group of turbines taking part in distribution is accepted as objective function

$$
q=\sum_{i=1}^{n} Q_{i}\left(\mathrm{D}_{P i}, \mathrm{D}_{H i}\right) / \sum_{i=1}^{n} N_{i}\left(\mathrm{D}_{P i}, \mathrm{D}_{H i}\right) \rightarrow \min
$$

a Corresponding author : bel@tpu.ru 


\section{MATEC Web of Conferences}

where - $Q_{i}\left(D_{P i}, D_{H i}\right)(i=1 \div n)$ - heat consumption for separate turbo-units depending on steam consumption in production $D_{P i}$ and heating $D_{H i}$ extractions according to their EC; $N_{i}\left(D_{P i}, D_{H i}\right)(i=1 \div n)$-Dependence of turbine electric capacity for heat consumption upon controlled load extractions.

Problem has limitations on steam consumption in heat-extraction of separate turbines.

$$
\begin{aligned}
& D_{P i}^{\min } \leq D_{P i} \leq D_{P i}^{\max }, i=1 \div n ; \\
& D_{H i}^{\min } \leq D_{H i} \leq D_{H i}^{\max }, i=1 \div n,
\end{aligned}
$$

in total load

$$
\begin{aligned}
& D_{P \Sigma}=\sum_{i=1}^{n} D_{P i} ; \\
& D_{H \Sigma}=\sum_{i=1}^{n} D_{H i} .
\end{aligned}
$$

Dynamic process of two-dimensional distribution of loads $D_{P \Sigma}$ and $D_{H \Sigma}$ between turbo-units of the group is introduced according to algorithm [4]. All the turbines from the group are numbered from 1 to $\mathrm{n}$ (randomly), this numbering to remain the same.

Every k step $(k=1 \div n)$ total thermal loads $D_{P \Sigma k}$ and $D_{H \Sigma k}$ change discreetly from minimum possible for this step $D_{P \Sigma k}^{\min }=\sum_{i=1}^{k} D_{P i}^{\min }$ and $D_{H \Sigma k}^{\min }=\sum_{i=1}^{k} D_{H i}^{\min }$ correspondingly, to maximum possible $D_{P \Sigma k}^{\max }=\sum_{i=1}^{k} D_{P i}^{\max }$ and $D_{H \Sigma k}^{\max }=\sum_{i=1}^{k} D_{H i}^{\max }$, where $\mathrm{k}$ - number of units participating in distribution. Besides there are discrete values, from $D_{P i}^{\min }$ to $D_{P i}^{\max }$ and from $D_{H i}^{\min }$ to $D_{H i}^{\max }$ correspondingly, of steam consumption in extraction of separated turbines $D_{P i}$ and $D_{H i}$. Change steps of production extraction are equal $\Delta D_{P}$, and heating - $\Delta D_{H}$.

Minimum specific heat consumption $q_{k}$ for the group consisting of $\mathrm{k}$ turbines from distribution of production $D_{P \Sigma k}$ and heating $D_{H \Sigma k}$ for k processes (heat extraction turbo-units) is calculated successively.

These values

$$
q_{k}\left(\mathrm{D}_{P \Sigma k}, \mathrm{D}_{H \Sigma k}\right)=\left[\sum_{i=1}^{k} Q_{i}\left(\mathrm{D}_{P i}, \mathrm{D}_{H i}\right) / \sum_{i=1}^{k} N_{i}\left(\mathrm{D}_{P i}, \mathrm{D}_{H i}\right)\right]=\min F_{k}\left(\mathrm{D}_{P 1}, \ldots, \mathrm{D}_{P k}, \mathrm{D}_{H 1}, \ldots, \mathrm{D}_{H k}\right),
$$

correspond to «conditionally optimal» mode of operation of a group consisting of k turbines with corresponding «conditionally optimal» energy characteristic $f_{Q_{k}}\left(D_{P \Sigma k}, D_{H \Sigma k}\right)$ and capacity of heat consumption $f_{N_{k}}\left(D_{P \Sigma k}, D_{H \Sigma k}\right)$ of turbines belonging to this group.

The algorithm includes two tasks: direct task allowing obtaining optimal solutions for k-step at any value of total loads and reverse as a result of which one can obtain optimal load of separate units at total loads.

Direct task includes stepwise building of functions according to recurrent Bellman correlations. Distribution of loads is reduced to solving of $\mathrm{n}$ two-dimensional problems by using the optimality principle.

The first step of multistage process includes building of functions for the first turbine at changing total values of $D_{P \Sigma}$ and $D_{H \Sigma}$ :

$$
\begin{aligned}
& f_{Q_{1}}\left(D_{P \Sigma}, D_{H \Sigma}\right)=Q_{1}\left(D_{P 1}, D_{H 1}\right) ; \\
& f_{N_{1}}\left(D_{P \Sigma}, D_{H \Sigma}\right)=N_{1}\left(D_{P 1}, D_{H 1}\right) ;
\end{aligned}
$$

where $D_{P 1}, D_{H 1}$ - designated with some steps $\Delta D_{P}$ and $\Delta D_{H}$ correspondingly, values of steam consumption in controlled extractions of the first turbine of the group.

It is obvious that the conditions are fulfilled

$$
\begin{gathered}
D_{P 1}=D_{P 1}^{\min } \div D_{P 1}^{\max } ; D_{H 1}=D_{H 1}^{\min } \div D_{H 1}^{\max } ; \\
D_{P \Sigma}=D_{P 1}^{\min } \div D_{P 1}^{\max } ; D_{H \Sigma}=D_{H 1}^{\min } \div D_{H 1}^{\max }
\end{gathered}
$$


Every next step has optimal distribution of loads $D_{P \Sigma}$ and $D_{H \Sigma}$ between k turbine and group of (k-1) turbines according to recurrent Bellman correlations. For this purpose optimization problems of minimizing specific heat consumption for the group of $\mathrm{k}$ turbines are solved.

$$
q_{k}\left(D_{P \Sigma}, D_{H \Sigma}\right)=\min F_{K}=\min \left[\frac{Q_{k}\left(D_{P k}, D_{H k}\right)+f_{Q_{k-1}}\left(\left(D_{P \Sigma}-D_{P k}\right),\left(D_{H \Sigma}-D_{H k}\right)\right)}{N_{k}\left(D_{P k}, D_{H k}\right)+f_{N k-1}\left(\left(D_{P \Sigma}-D_{P k}\right),\left(D_{H \Sigma}-D_{H k}\right)\right)}\right]
$$

Heat extraction parameters of extraction of $\mathrm{k}$ turbine are changed within the control range $D_{P k}=D_{P k}^{\min } \div D_{P k}^{\max } ; D_{H k}=D_{H k}^{\min } \div D_{H k}^{\max }$ on this step according to limitations, total load are running the values

$$
\begin{aligned}
& D_{P \Sigma k}=\left(\sum_{i=1}^{k} D_{P k}^{\min }\right) \div\left(\sum_{i=1}^{k} D_{P k}^{\max }\right) ; D_{H 1}=D_{H 1}^{\min } \div D_{H 1}^{\max } \\
& D_{H \Sigma k}=\left(\sum_{i=1}^{k} D_{H k}^{\min }\right) \div\left(\sum_{i=1}^{k} D_{H k}^{\max }\right) ; D_{H 1}=D_{H 1}^{\min } \div D_{H 1}^{\max } .
\end{aligned}
$$

Solution of problem (7) allows finding «conditionally optimal» energy characteristic of turbines of this group (numerator of expression (7)

$$
f_{Q_{k}}\left(D_{P \Sigma}, D_{H \Sigma}\right)=Q_{k}\left(D_{P k}, D_{H k}\right)+f_{Q_{k-1}}\left[\left(D_{P \Sigma}-D_{P k}\right),\left(D_{H \Sigma}-D_{H k}\right)\right]
$$

and their «conditionally optimal» heat consumption capacity (denominator)

$$
f_{N_{k}}\left(D_{P \Sigma}, D_{H \Sigma}\right)=N_{k}\left(D_{P k}, D_{H k}\right)+f_{N_{k-1}}\left[\left(D_{P \Sigma}-D_{P k}\right),\left(D_{H \Sigma}-D_{H k}\right)\right] \text {. }
$$

The obtained functional dependencies (8) and (9) are used at the next $(\mathrm{k}+1)$ step of optimization.

According to the above-mentioned, the accepted optimization criterion (1) has changed the recurrent Bellman formula as compared to its traditional representation, the essence of functional dependence considering «conditionally optimal» solutions of previous optimization steps to remain unchanged.

Reverse problem is reduced to sequential use of functions $f_{Q_{k}}\left(D_{P \Sigma}, D_{H \Sigma}\right)$ and $f_{N_{k}}\left(D_{P \Sigma}, D_{H \Sigma}\right)$ at changing $\mathrm{k}$ from $\mathrm{n}$ to 1 .

\section{Results}

As the example of successful application of two-dimensional dynamic modeling body of mathematics one can consider the solving of problem of thermal load distribution on one Heat station of the Siberian region [5], which has heat-extraction turbo-units of different types: T-25-90, PT-25-90, K-50-90-2M, KT-100-90, P-12-90/18. Heat distribution from the station is performed as steam of two pressures, 1,6 and 1,0 MPa, and hot water from three boilers with its own temperature graphs. Complex system of limitations considers the distribution of steam from producing and heating extractions of turbines in general stations collectors of different pressures as well as peculiarities of main and peaking hot-water boiler steam consumption. One can see on Fig 1 the results of optimal load of heating extractions of turbines at given system parameters of hot-water supply depending on ambient air temperature $t_{\text {a.t. }}$. 


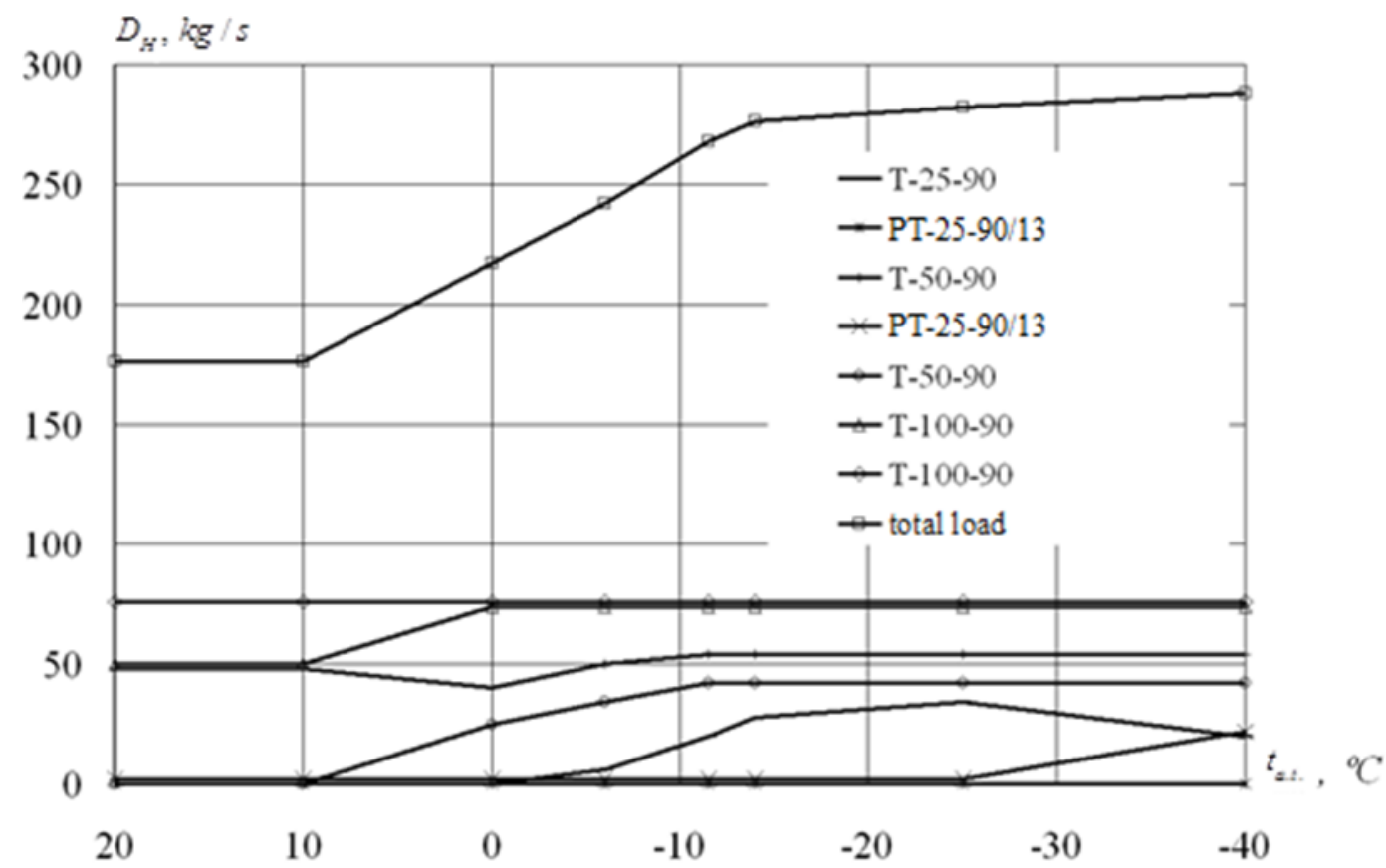

Figure 1. Optimal load of heating extractions of HES turbines $=9,0 \mathrm{MPa}$, depending on ambient air temperature

\section{Conclusion}

1. The algorithm of two-dimensional dynamic distribution of thermal loads between heating and production extractions of heat-extraction turbines is developed

2. Selected as optimization criterion, specific heat consumption for a group of turbines changes the recurrent Bellman correlation as compared to its classic expression.

3. Algorithm operability is proved by means of developed program complexes for optimization of modes of operational heat stations.

The work is completed using the funds of the Competitiveness Enhancement Program of TPU (Project_Leading Research University_Institute of Power Engineering_138_2014).

\section{References}

1. Kachan A. D., Optimization of modes and increase of efficiency of steam-turbine plants of TPS (Higher school, Minsk, 1985)

2. Shisheya P.N. Research of heat-extraction steam-turbine plants and development of methods of in-office optimization of their operation. Ph.D. Abstarct of a thesis (Belarus Polytechnic University, Minsk, 1980)

3. Belyaev L.A., Romasheva O.Y. Collected papers of X Russian scientific and technical conference, 189 (2004)

4. Bellman R., Dreyfus S. Dynamic programming applied problems (Moscow, Nauka, 1968)

5. Belyaev L.A., Romasheva O.Y., Volkov V.N. Collected papers of XII Russian scientific and technical conference «Energetics: Ecology, safety, reliability» (2006) 